\title{
Estimation of QoE of Video Traffic using a Fuzzy Expert System
}

\author{
${\text { Jeevan } \text { Pokhrel }^{* \dagger} \text {, Bachar Wehbi*,Anderson Morais }{ }^{\dagger} \text {, Ana Cavalli }{ }^{\dagger} \text {, Eric Allilaire }}^{\ddagger}$ \\ *Montimage, 39 rue Bobillot, 75013, Paris, France \\ †Telecom SudParis, Evry, France \\ $\ddagger$ Vierling Communication SAS Plaisir, France \\ \{jeevan.pokhrel, bachar.wehbi\}@montimage.com \\ \{anderson.morais, ana.cavalli\}@it-sudparis.eu \\ eric.allilaire@vierling-group.com
}

\begin{abstract}
Quality of experience (QoE) in multimedia traffic has been the focus of extensive research in the last decade. The estimation of the $\mathrm{QoE}$ provides valuable input in order to measure the user satisfaction of a particular service. QoE estimation is challenging as it tries to measure a subjective metric where the user experience depends on a number of factors that cannot simply be measured. In this work, we present a methodology and a system based on fuzzy expert system to estimate the impact of network conditions $(\mathrm{QoS})$ on the QoE of video traffic. At first, we conducted subjective tests to correlate network QoS metrics with participants' perceived QoE of video traffic. Second, we propose a No Reference method based on fuzzy expert system to estimate the network impact on the video QoE. The membership functions of the proposed fuzzy system are derived from normalized probability distributions correlating the QoS metrics with QoE. We propose a simple methodology to build the fuzzy inference rules. We evaluated our system in two different sets of experiments. The estimated video quality showed high correlation with the subjective QoE obtained from the participants in a controlled test. We integrated our system as part of a monitoring tool in an industrial IPTV test bed and compared its output with standard Video Quality Monitoring (VQM). The evaluation results show that the proposed video quality estimation method based on fuzzy expert system can effectively measure the network impact on the QoE.
\end{abstract}

Index Terms-fuzzy expert system, quality of experience, traffic monitoring, video.

\section{INTRODUCTION}

Internet traffic is growing exponentially due to the advances in technologies, the higher achievable bit rates and the proliferation of smart phones with their applications ecosystem. Video traffic is expected to reach $66 \%$ of the global mobile traffic by the year 2015 with one million minutes of video content crossing the Internet every second [1]. This dominance in itself illustrates the importance of estimating the satisfaction of users when watching an Internet video clip. Although web video (e.g., YouTube) is responsible for most of the video traffic, VoD and IPTV services are expected to generate more revenues for the network operators and service providers. The fast growth in the consumption of internet videos shows that IPTV has a very promising future as a way of providing video content to end-users via television and mobile [2].

According to the ITU-T Focus Group on IPTV [3] Quality of Experience (QoE) refers to "the overall acceptability of an application or service, as perceived subjectively by the enduser". Therefore, QoE is a subjective measure and can vary according to the user expectation and context. Moreover, it is an overall end to end system effect (client, terminal, network, services infrastructure, media encoding, etc.) and depends on a number of factors that cannot simply be measured. It requires tests with actual users in a controlled environment to properly estimate the QoE; which is costly and time consuming. However, service providers and network operators are more interested in tools that can objectively reflect the subjective mean opinion score of users, with reasonable accuracy.

The ability to identify the perceived degree of video impairment due to network perturbation is a key point in the quality estimation of video traffic. Moreover, the effect of network perturbations on video can range from distortionless to intolerable distortion. Therefore, measuring the impact of network perturbation on quality of the video traffic is a challenging task as shown in several works [4] [5] and [6].

In this paper, we propose a QoE estimation system based on fuzzy logic [7] to estimate the impact of the network conditions on the video quality, i.e., the QoE. We consider three QoS metrics (packet loss rate, packet loss burstiness, and, jitter) as the network condition indicators. The variation of these QoS metrics impacts the quality of the delivered video and, consequently, the user satisfaction level. Our objective is to design and implement a method to estimate the variation of the user satisfaction level in function of the network QoS conditions. At first, we perform a set of subjective tests with real participants to measure the correlation between QoS metrics and QoE of video traffic. Second, we propose a fuzzy expert system which is based on No Reference method that can estimate the video quality based on the network conditions. The correlation between the QoS metrics and the participants QoE is transformed into fuzzy membership functions using probability distribution functions and curve fitting methods. We also propose a simple methodology for fuzzy inference rules generation by assigning weights to the video impairment scores. Two different sets of experiments were performed to evaluate our system. In the first, we simulated our system in MATLAB and compared the estimated QoE output (called also estimated MOS or eMOS) with the 
subjective QoE obtained from the participants in a controlled test. This experiment validated our methodology showing high correlation between our estimated and the subjective QoE. In the second experiment, we integrated our system as part of a monitoring tool in an industrial IPTV test bed and compared its output with standard Video Quality Monitoring (VQM). The outputs of both video quality estimation methods were also correlated. The experiment results show that the proposed video quality estimation method based on fuzzy expert system can effectively measure the network impact on the QoE.

The rest of the paper is organized as follows. Section II describes the related work. In section III, the methodology for video quality estimation is illustrated. In section IV, we validate our methodology and evaluate it in an industrial IPTV test bed. Finally, section V gives a conclusion and directions for future work.

\section{RELATED WORKS}

Quality estimation of video traffic can be subjective or objective. Subjective methods consist of many users viewing sample multimedia and rating its quality according to predefined quality scale depending on their personal perception. Objective methods try to measure the QoS based on objectively measured network or media metrics. Objective methods can be divided into full reference (FR), no reference (NR) and partial reference (PR). In full reference methods, the distorted sample is compared with the original sample including perpixel processing and temporal/spatial alignment. No reference methods only use the degraded signal for the quality estimation and have no information about the original reference sequence. Partial reference methods lie between FR and NR methods. They are designed to predict the perceptual quality with only partial information about the reference sequence.

\section{A. Subjective Video Quality Estimation}

One of the most popular subjective video quality test methods is Mean Opinion Score (MOS) [8]. It was designed for testing the speech quality but, nevertheless, has become a common method for evaluating multimedia traffic. Double Stimulus Continuous Quality Scale (DSCQS) [9] and Double Stimulus Impairment Scale (DSIS) [9] are other two similar methods for subjective video quality evaluation. However, they are not relevant for assessing the quality of long video sequences. Also, they were not designed for the quality evaluation of video transmission over packet networks, like the Internet, because of its non-deterministic behavior and the bursty nature of encoded video. Similarly, Single Stimulus Continuous Quality Scale (SSCQE) [9] evaluates the video quality instantaneously by continuously adjusting a slider from bad to excellent. These methods require appropriate testing environment and strict attention. In addition, subjective quality estimation processes are costly, time consuming and hard to automate. Objective methods try to overcome these limitations by providing mathematical calculation for the quality estimation making them adequate for network quality monitoring.

\section{B. Objective Video Quality Estimation}

Peak Signal to Noise Ratio (PSNR) is one of the most popular objective video quality estimation methods. It is computationally fast and mathematically simple to understand. However, it poorly correlates with the human perception of visual quality [10]. Video Quality Model (VQM) [11] measures the perceptual effects of video impairments including blurring, jerky/unnatural motion, global noise, block and color distortion; and, combines them into a single metric. Perceptual Video Quality-of-Experience Measurement (PEVQ) [12] performs signal analysis to evaluate the video quality. It is a referenced based method where a degraded video signal is compared to the original signal on a perceptual basis. However, measuring perceptual effect of video impairment is complex. Media Delivery Index (MDI)[13] is a scalable metric for assessing the effect of delivery network on the video and can be measured from any point between the video end points. It has two components, delay factor (DF) and media loss rate (MLR), both using packet loss and jitter as predictors of video quality.

Our objective is to develop a video quality estimation system that requires no reference video, but maps QoS properties of video traffic into user perceived QoE in order to measure the impact of the network on the delivered video quality. In this work, we model a subjective test result with a fuzzy expert system and used this model as objective tool to measure the impact of the network on the delivered video quality. An advantage of fuzzy expert system is that they are simple and computationally less intensive.

\section{Methodology}

In order to develop our video quality estimation technique, we followed a methodology that consists on conducting subjective tests with end user participants in order to build a learning set for correlating objective network QoS metrics with the subjective QoE provided by the participants. This correlation was then used to build the membership functions and inference rules of our fuzzy expert system for video QoE estimation.

\section{A. Subjective Tests}

In the subjective test, we presented different video clips to the 25 participants who rated each video clip according to the perceived impairment giving one of the following scores:

- Imperceptible (score 5)

- Perceptible but not annoying (score 4)

- Slightly annoying (score 3)

- Annoying, and (score 2)

- Very annoying (score 1)

We used six video clips of different types (sports, movie, animation, and interview) and generated 228 sample video clips which were constructed with different network level perturbations. We constructed these video clips by streaming from a server to client and correspondingly introducing perturbation through emulated network. We selected three QoS parameters for perturbation; packet loss, jitter and packet loss burstiness, 
which we considered promising for the mapping of QoS to QoE for video traffic. These constructed video clips along with the original video clips were shown to different participants in a random order in a closed room. For each participant it took around 2.5 hours to perform the test. After watching 20 video clips a pause of 5 minutes was taken. Figure 1 illustrates the experimental environment for the subjective tests.

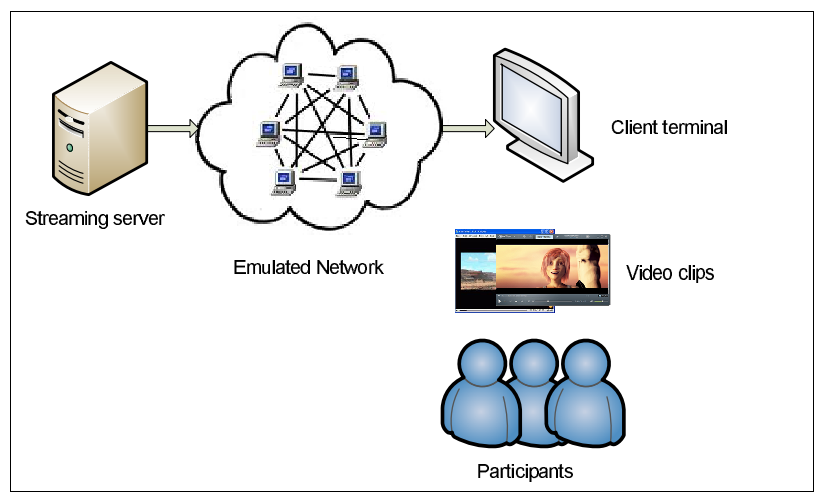

Fig. 1. Experimental setup for subjective tests.

\section{B. Correlation between QoS Metrics and QoE}

From the subjective test, we built a learning set that consisted of the mapping between the participants' scores and the QoS metrics for each of the considered video clips. We used a probabilistic approach to correlate QoS metrics to the participants' scores. Therefore, for every QoS metric, we built five different probability distribution functions (pdf) (one function per QoE score) that provide the variation of the participants' ratio (\%) with the QoS metric for a specific QoE score. This probabilistic information was changed into a fuzzy set by dividing the pdf by its peak value (normalized pdf) [14]. The fuzzy set, which has the same form as that of the original pdf, is converted into an equivalent triangular or trapezoidal fuzzy set by using a curve fitting method [15]. The triangular or trapezoidal fuzzy set represents the membership functions for the different QoS metrics.

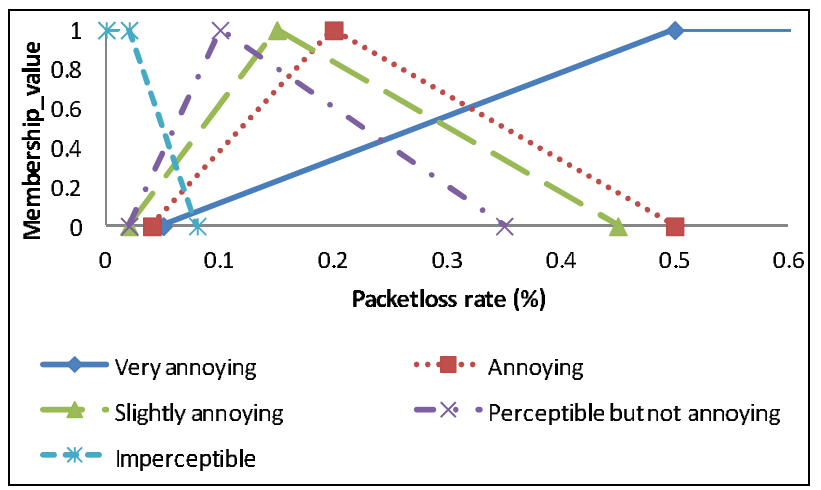

Fig. 2. Membership functions for packet loss rate metric.

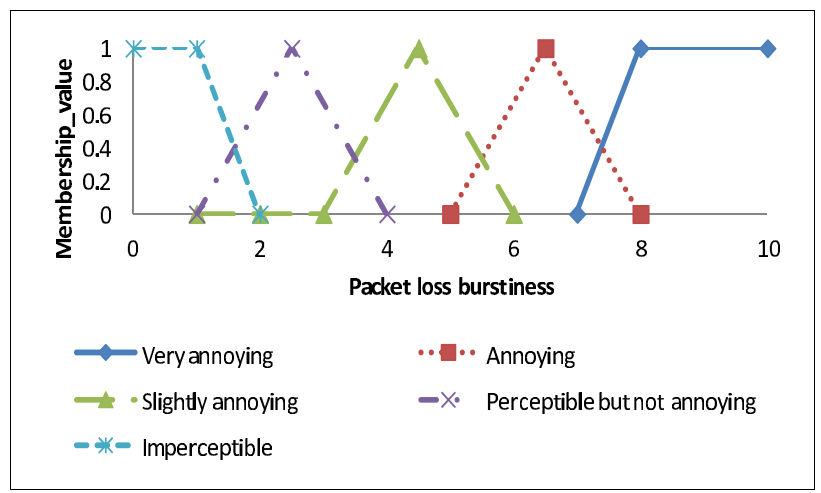

Fig. 3. Membership functions for packet loss burstiness metric.

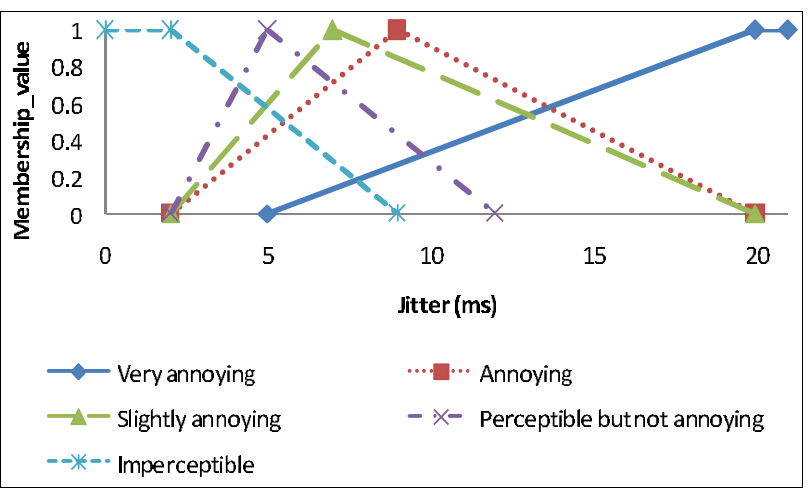

Fig. 4. Membership functions for jitter metric.

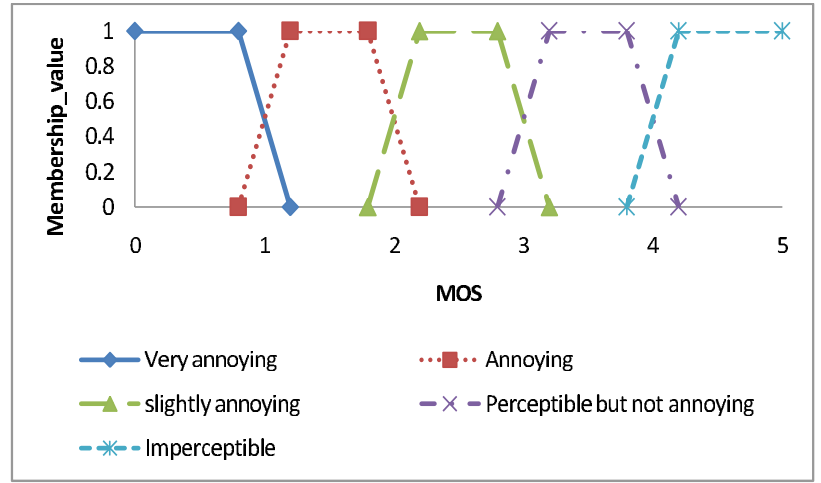

Fig. 5. Membership function for the estimated MOS (eMOS).

Figure 2 illustrates the QoE scores membership functions associated with the packet loss rate QoS metric. For example, the packet loss rate of $0.2 \%$ has membership values of $0,0.6$, $0.8,1$ and 0.35 corresponding respectively to the QoE scores $5,4,3,2$, and, 1 . We note that a membership value of 1 represents a high degree of membership to the corresponding class and decreasing membership value represents deviation from the class. Figure 3 and Figure 4 illustrate the membership functions for packet loss burstiness and jitter metrics respectively. Similarly, in Figure 5, the membership functions for the estimated QoE are defined according to the standard MOS [8] definition. 


\section{Video Quality Estimation Fuzzy Inference Rules}

The correlation between the QoS metrics and the video quality, described in the subsection III.B, allowed to build five fuzzy membership functions for the three considered QoS metrics (packet loss rate, packet loss burstiness and jitter). Based on the combinations of QoS metrics and their rating, we have to estimate whether the network impact on the video quality (QoE) is imperceptible (excellent conditions), perceptible but not annoying (good conditions), slightly annoying (fair conditions), annoying (poor conditions) or very annoying (bad conditions). That is, we need to associate an estimated QoE score for the different combination of QoS metrics scores. For example; IF (Packet loss is very annoying) \& (Burst Loss is very annoying) \& (Jitter is very annoying) THEN (the estimated QoE is very annoying).

Considering the combination of the QoS metrics scores, we have a set of $5^{3}$ possible rules. We follow the following methodology to define the rules while at the same time reducing their number. We associate a "weight" to each rating as follows: 0 for "imperceptible", 1 for "perceptible but not annoying", 3 for "slightly annoying", 5 for "annoying", and, 7 for "very annoying". For every combination, we calculate the rule weight as the sum of the weights of the QoS metric scores. The rule output corresponds to the estimated QoE score as defined in TABLE I. For instance, if all the QoS metrics scores are imperceptible then rule score is $0(0+0+0)$, which corresponds to "imperceptible" QoE score. Likewise, if two QoS metrics scores are "perceptible but not annoying" and one is "slightly annoying", then the rule weight is $5(1+1+3)$, which corresponds to an "annoying" QoE score.

TABLE I

RULE WEIGHT TO QOE SCORE MAPPINGS

\begin{tabular}{|l|l|}
\hline Rule weight & Estimated QoE score \\
\hline 0 & Imperceptible \\
\hline $1-2$ & Perceptible but not annoying \\
\hline $3-4$ & Slightly annoying \\
\hline $5-6$ & Annoying \\
\hline $7+$ & Very annoying \\
\hline
\end{tabular}

\section{QoE Estimation System}

Our proposed video QoE estimation system is based on fuzzy logic that is powered with a learned membership functions (QoS/QoE correlation) and a set of fuzzy inference rules. Fuzzy logic is a well-known technique that can handle problems with imprecise and incomplete data[7]. Figure 6 illustrates the building blocks of our proposed system that can be placed at any point in the network between the video source and the terminal. The system performs a per-flow analysis. A Deep Packet Inspection (DPI) engine at the entry point of the system inspects the network traffic to identify video flows and extract relevant per-flow QoS metrics (packet loss rate, packet loss burstiness and jitter). These metrics are constantly fed to the fuzzy expert system that uses the defined membership function and inference rules to estimate the QoE on a per flow basis.

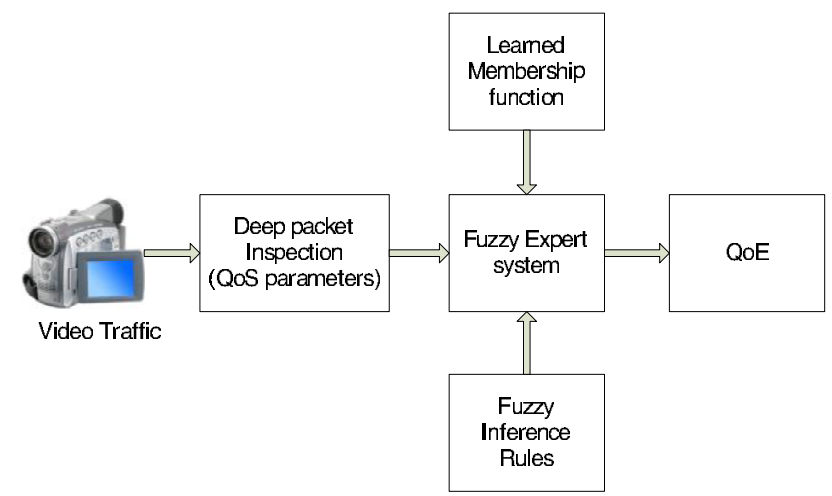

Fig. 6. Membership function for the estimated MOS (eMOS).

\section{VALIDATION AND EXPERIMENTAL RESULTS}

\section{A. Validation of the Proposed Methodology}

To validate the proposed methodology, we compared the results obtained from the subjective tests (see section III.A) with those obtained from our proposed system. For this end, we used the Fuzzy logic toolbox of MATLAB [16] and developed a simulation scenario with our membership function and rules for validation. Video clips with different QoS metric values were used for validation.

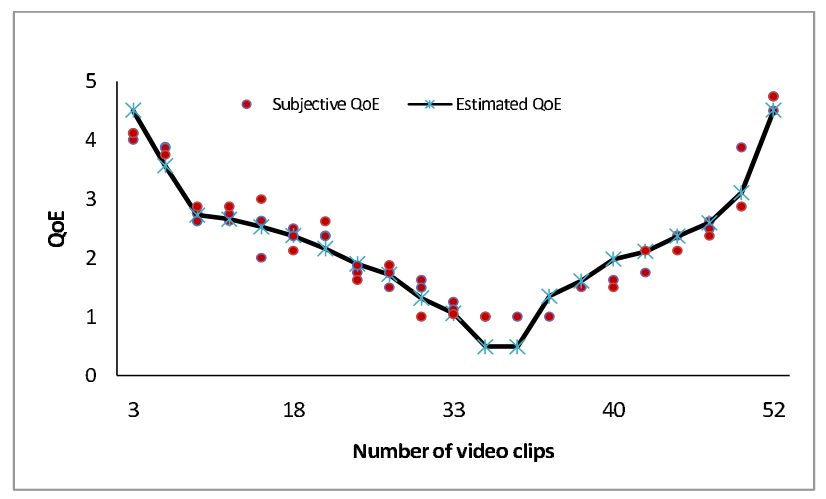

Fig. 7. Comparison between the subjective and estimated MOS.

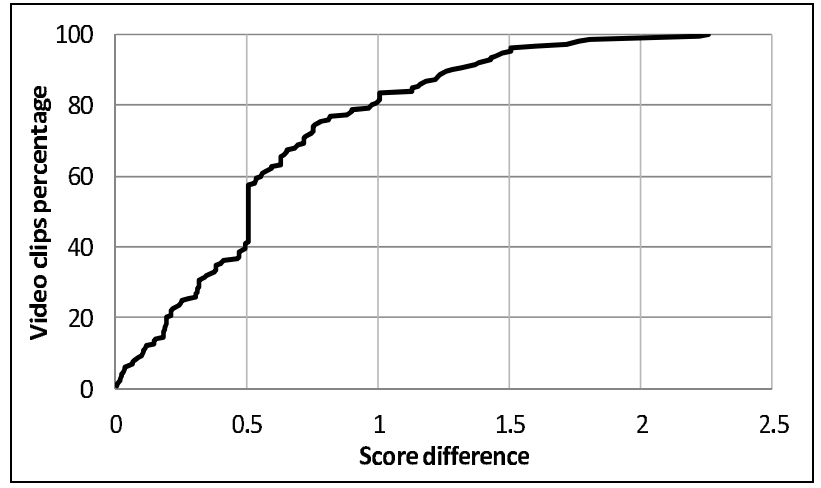

Fig. 8. Probabiltiy distribution of the subjective and estimated MOS difference.

For each video clip, we obtained subjective QoE from subjective tests as described in section III.A; and, estimated 
QoE from our system simulated in MATLAB. Each point in Figure 7 represents the subjective MOS of a particular video clip and the line represents the estimated QoE. It shows that the estimated QoE is highly correlated with the subjective results. This indicates that the proposed system succeeds in reflecting the users perception. This is also illustrated in Figure 8 that considers the probability distribution of the difference between the participants subjective scores (MOS) and the estimated scores. We can see that in around $60 \%$ of the tests the score differences were less than 0.5 . It reached $83 \%$ for score differences less than 1. This means that in $83 \%$ of the cases, the differences in the subjective and estimated QoE were at most one score level (e.g., if the participants reported "Imperceptible" video quality, our method would have reported either "imperceptible" or "perceptible but not annoying". Only $4 \%$ of the tests showed a score differences of more than 1.5. This estimation accuracy emphasizes the ability of the proposed system to measure the impact of the network conditions on the user satisfaction.

\section{B. Real Test Bed Experimentations}

To evaluate our system in close to real network conditions, we implemented the proposed fuzzy expert system for video quality estimation as a module in a MMT (Montimage Monitoring Tool) [17] probe. Then we integrated MMT probe and a VQM probe in an industrial IPTV test bed [18]. The experiments consisted in comparing the results of both probes in the presence of different emulated IP level and copper line perturbations. In the following sections, we first describe the industrial evaluation test bed then we present an analysis of the experimental results.

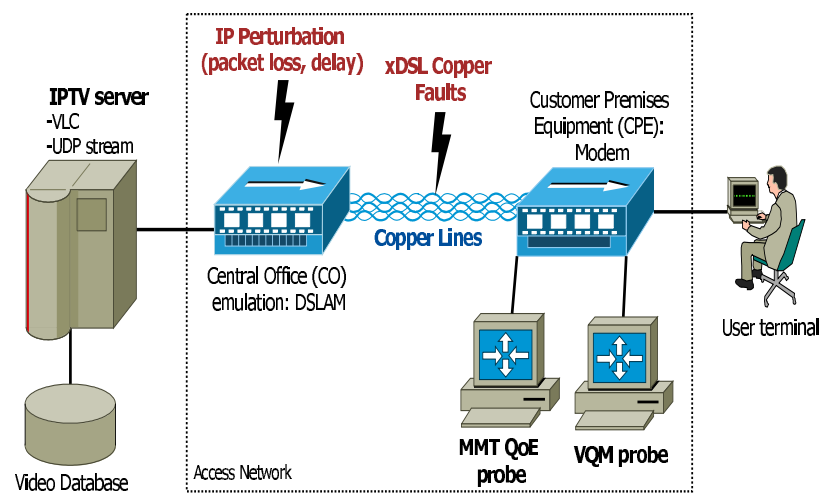

Fig. 9. Vierling experimental test bed for QoE measurements.

1) Test Bed for QoE Measurement: Figure 9 presents the building blocks of Vierling experimental test bed, which is an industrial evaluation test bed for $\mathrm{QoE}$ assessment in Digital Subscriber Line technology (xDSL).

The test bed is constituted of an IPTV server that uses VLC to stream video clips over RTP/UDP transport. The streaming server is connected to a Digital Subscriber Line Access Multiplexer (DSLAM) modem emulator at the central office (CO). At this point of the network, an IP traffic perturbation tool is used to emulate network conditions by introducing a variety of network impairments such as packet loss, delay, and jitter. These perturbations are used for quantifying the network impact on the video QoE. The DSLAM modem is connected via kilometers length copper lines to the Customer Premises Equipment (CPE) xDSL modem. Furthermore, the test bed allows performing measurements on multiple copper line configurations at the network access level and allows adding xDSL disturbers with the help of remote controlled HF signal and noise generator.

The test bed can reproduce a typical DSL network configuration: (i) the IPTV server represents the service provider network, and (ii) the DSLAM modem emulator and CPE (Customer Premises Equipment) modem correspond to the access network on a physical copper line with thousands of copper configurations.

In addition, external network probes can be attached to the modem at the CPE side to sniff the video traffic and analyze the collected data in order to correlate QoS parameters and estimate the corresponding QoE level.

We implemented our QoE estimation system as a module in a MMT to facilitate its deployment in IP based networks. Now MMT method refers to our QoE estimation method. This monitoring tool uses DPI to detect video flows and extract the QoS metrics of interest to be used for the QoE estimation.

At the CPE side, we installed MMT probe along with a VQM probe in order to compare the results of the two methods. The QoE measurements consisted in analyzing the network traffic of the broadcasted video clips. Two different types of deterioration factors were simulated: xDSL noise and copper faults on the copper cables, and, packet loss, and message delay/jitter on the IP level on the DSLAM modem.

We compare the results of the MMT method with those obtained using the VQM method.

2) Experimental Results: A total number of around 600 video streams were analyzed. For every stream, we collected the applied perturbation type, the QoS parameters, and the estimated MOS given by the MMT method and by the VQM method.

Figure 10 illustrates the variation with the packet loss rate of the estimated Mean Opinion Score (eMOS) given by MMT and by VQM. We can see that the eMOS values decrease with the packet loss increase for both methods. The decrease, however, is sharper using MMT method. This is due to the fact that MMT estimation model is mainly designed for high definition video quality. In fact, the subjective test described in section III.A used exclusively HD video clips. In the test bed experiments, average quality videos were used. This fact made the transmitted video clips less sensitive to packet loss than HD videos. At very low packet loss rate (less than $0.08 \%$ ), the eMOS of MMT is higher than that of VQM. This is because the VQM method takes into account the quality of the original video while MMT systems objective is to measure the impact of the network on the transmitted video quality. MMT higher eMOS score means that the network conditions are favorable. As the packet loss rate increases, the eMOS of both methods decrease to reach a point where the estimated quality becomes 
very bad. We should note here that MMT systems eMOS has a maximum value of 4.51 and a minimum value of 0.494 . This is due to the centroid method used for defuzzification in the fuzzy expert system [7].

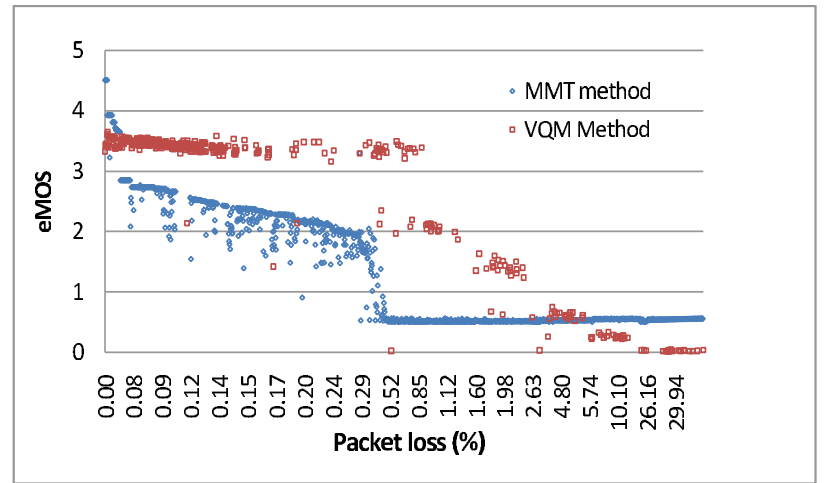

Fig. 10. Variation of eMoS with the packet loss rate.

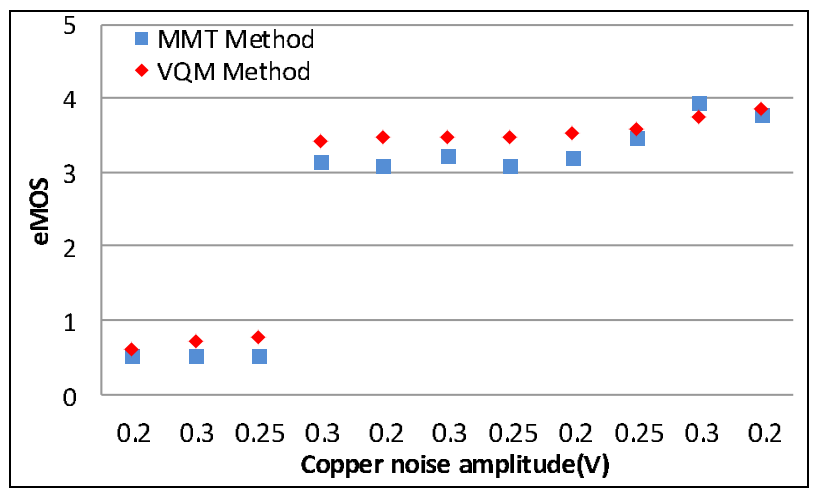

Fig. 11. Estimated MoS in different copper line perturbations.

Figure 11 compares the results of MMT method and VQM method for different copper line perturbations (noise of different amplitudes). We can see that the results of both methods are highly correlated. The results of the test bed evaluation show that MMT proposed video quality estimation succeeds in reflecting the impact of network perturbations on the video quality.

\section{Conclusions}

In this paper, we have proposed a video quality estimation method based on a fuzzy expert system to measure the impact of network condition (QoS parameters) on the user perceived satisfaction level (QoE) of video services. We have performed a set of subjective tests with real participants in order to correlate network QoS parameters levels with the user perceived video quality. The defined fuzzy membership functions were derived from the QoS/QoE correlation using probability distribution functions. We have also proposed a simple method to build estimation inference rules by assigning weights to the different video impairment scores. The proposed methodology has been validated against the results of subjective tests. The proposed system has been integrated and tested in an industrial IPTV evaluation test bed. The validation and experimental results have shown that our QoE estimation method is highly correlated to both the participants subjective QoE scores as well as to the estimated MOS given by the VQM method. As future work, we envisage to extend our approach by adding additional analysis parameters to the system, such as, video codec and bit rate. We will also investigate the usage of the feedback of our system to implement corrective actions on network and application levels to recover the QoE to satisfactory levels, e.g., adjusting the video transmission rate.

\section{Acknowledgements}

This work has been carried out in the framework of European research initiative CELTIC-Plus of EUREKA ICT Cluster in the context of project $\mathrm{CP}$ 7-009 IPNQSIS and project CP7$011 \mathrm{MEVICO}$.

\section{REFERENCES}

[1] "Cisco visual networking index: Forecast and methodology, 2010 2015," whitepaper, 2011.

[2] M.-J. Montpetit, N. Klym, and T. Mirlacher, "The future of iptv," Multimedia Tools and Applications, vol. 53, pp. 519-532, 2011.

[3] "Quality of experience requirements for iptv services," ITU-T Recommendation G.1080, 2008.

[4] S. Winkler and R. Campos, "Video quality evaluation for internet streaming applications," Human Vision and Electronic Imaging, vol. 3, pp. 104-115, 2003.

[5] D. Wijesekera, J. Srivastava, A. Nerode, and M. Foresti, "Experimental evaluation of loss perception in continuous media," 1999.

[6] O. Issa, F. Speranza, W. Li, and H. Liu, "Estimation of time varying qoe for high definition iptv distribution," in Consumer Communications and Networking Conference (CCNC), 2012 IEEE, jan. 2012, pp. 326 -330.

[7] M. Negnevitsky, "Artificial intelligence, a guide to intelligent system," Addision-Weslay, 2002.

[8] "Mean opinion score (mos) terminology," ITU-T Recommendation P.800.1, 2006.

[9] "Methodology for the subjective assessment of the quality of television pictures," ITU Recommendation BT.500-11, 2002.

[10] "Objective perceptual video quality measurement techniques for digital cable," ITU-T Recommendation J.144, 2003.

[11] M. Pinson and S. Wolf, "A new standardized method for objectively measuring video quality," IEEE Transaction Broadcasting, vol. 50, pp. 312-322, 2004.

[12] "Objective perceptual multimedia video quality measurement in the presence of a full reference," ITU Recommendation J.247,, 2008.

[13] "Whitepaper: Iptv qoe: Understanding and interpreting mdi values," Agilent technologies, 2008.

[14] B. S. de Lima and N. F. Ebecken, "A comparison of models for uncertainty analysis by the finite element method," Finite Elements in Analysis and Design, vol. 34, no. 2, pp. $211-232,2000$.

[15] M. Anoop, K. B. Rao, and S. Gopalakrishnan, "Conversion of probabilistic information into fuzzy sets for engineering decision analysis," Computers \&amp; Structures, vol. 84, pp. 141-155, 2006.

[16] MATLAB, "Fuzzy logic toolbox." [Online]. Available: http://www.mathworks.fr/products/fuzzy-logic

[17] "Montimage monitoring tool (mmt)," http://www.montimage.com/products.html, 2012.

[18] A. Cuadra-Sanchez, "D2.2. definition of requirements of the management systems to keep up with qoe expectations based on qos and traffic monitoring." [Online]. Available: http://ipnqsis.org/index.php?title=Deliverables 